\title{
Study on optimum synthesis of ethyl cyanoacetate
}

\author{
CHEN Hong $^{1}$, ZHU Bao-wei ${ }^{1}$, LIANG Hai ${ }^{1}$, YANG He² ${ }^{2}$ WANG Hao-yi ${ }^{1}$ \\ ${ }^{1}$ Chemical and environmental Engineering Department, Yingkou Institute of Technology, Yingkou, 115014, China \\ ${ }^{2}$ Liaoning Yinzhu Chemtex Group co., LTD, Yingkou, 115000, China
}

\begin{abstract}
Ethyl cyanoacetate is an important chemical intermediate and has been used widely. Ethyl cyanoacetate was prepared by esterification of cyanoacetic acid and absolute ethanol with mixed catalyst (the same proportion of silicotungstic and p-toluene sulfonic acid as catalyst). The influence factors of the amount of catalyst, $\mathrm{n}$ (cyanoacetic acid): $\mathrm{n}$ ( absolute ethanol), reaction time and reaction temperature on the esterification rate was investigated by orthogonal experiment of four factors and three levels. The product was analyzed by gas chromatography to determine the optimum reaction conditions.
\end{abstract}

\section{Introduction}

Ethyl cyanoacetate molecular formula is $\mathrm{C}_{5} \mathrm{H}_{7} \mathrm{NO}_{2}$ and structural formula is $\mathrm{NCCH}_{2} \mathrm{COOCH}_{2} \mathrm{CH}_{3}$. Ethyl cyanoacetate is an intermediate of fine chemical products such as medicine and dyes [1]. It is also used in the synthesis of esters, amides, acids and nitriles [2]. It is an intermediate for the preparation of 2-amino-4, 6dimethoxypyrimidine, which can be prepared as an intermediate for sulfonylurea herbicides, as well as an intermediate for the insecticide flufenitrile [3]. There is a wide range application prospects for ethyl cyanoacetate[4].

At present, the main methods for preparing ethyl cyanoacetate are as follows:Ethyl chloroacetate cyanidation method, methyl cyanoacetate transesterification method,cyanoacetate esterification method etc.are used frequently. Catalysts are important in the synthesis of ethyl cyanoacetate, such as heteropoly acid. Heteropoly acid, as a catalyst with less environmental pollution, there is strong acidity and oxidation reducibility, and the advantages of stable structure, high activity, non-corrosive equipment, little pollution, etc. [5]. Heteropoly acid is a good catalyst in esterification reaction.

Silicotungstic acid was used in this experiment. Ethyl cyanoacetate was prepared by esterification of cyanoacetic acid and absolute ethanol. The factors influencing the esterification rate, such as catalyst dosage, reaction time, reaction temperature, were analyzed. Optimization conditions were obtained through orthogonal experiment.

\section{The experiments}

\subsection{Materials}

Cyanoacetic acid (Yingkou sanzheng Fine Chemical Co., LTD.), absolute ethanol, silicotungstic acid, p-toluene sulfonic acid (Tianjin Zhiyuan Chemical Reagent Co., LTD.), phosphomolybdic acid, phosphotungstic acid (Sinopac Chemical Reagent Co., LTD.), 99\% ethyl cyanoacetate, benzene (Alighting Reagent Co., LTD.)

\section{2.apparatus}

Main experimental instruments required for the experiment :JJ-1 Precision electric mixer (Zhengzhou Ganzheng Equipment Co., LTD.), SP2100A gas chromatograph (Beijing Beifen-Ruili Analytical Instrument Group Co.,Ltd.), etc.

\subsection{Experiment methods}

The device used in this experiment is shown in Figure 1.

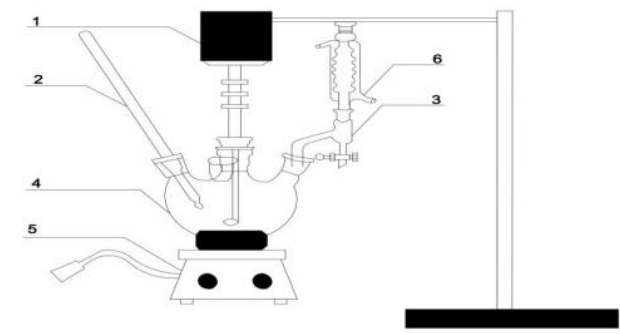

Fig. 1 Reaction device diagram

1-electric agitator 2 -thermometer 3-water konckout vessel 4four-necked flask 5-heating device

In this experiment, ethyl cyanoacetate was prepared by esterification of cyanoacetic acid and absolute ethanol. Cyanoacetic acid, absolute ethanol and catalyst were added into four-necked flask and stirred with an electric agitator to make the evaporated water and absolute ethanol flow back to the water separator through the condensing pipe.Gas chromatography analyzer was used for analysis and internal standard method was used for calculation.

\footnotetext{
* Corresponding author:chh021567@126.com
} 


\section{Results and discussion}

The catalyst was examined firstly, then the single factor experiments and orthogonal experiment were carried out, and the results were analyzed to determine the optimum reaction conditions.

In this experiment, benzene was used as the internal standard, 99\% pure ethyl cyanoacetate was used as the reference substance. The correction factor was calculated, and then the correction factor was used to calculate the content of ethyl cyanoacetate prepared.

Gas chromatography conditions: The column is KB-1, the carrier gas is nitrogen, the detector is FID, and the temperature is programmed. The inlet temperature is $240{ }^{\circ} \mathrm{C}$, the initial temperature of the column box is $120^{\circ} \mathrm{C}$, and the heating rate is increased at $30^{\circ} \mathrm{C} / \mathrm{min}$. The temperature was maintained at $205^{\circ} \mathrm{C}$ for 5 minutes and the detector temperature was $255^{\circ} \mathrm{C}$.

The calculation formula of correction factor 1 is as follows:

$$
\mathrm{f}=\frac{\mathrm{m}_{\mathrm{r}} / \mathrm{m}_{\mathrm{s}}}{A_{r} / A_{s}}=\frac{m_{r}}{m_{s}} \times \frac{A_{s}}{A_{r}}
$$

$\mathrm{m}_{\mathrm{s}}$-the mass of the internal standard benzene $\mathrm{m}_{\mathrm{r}}$-the quality of $99 \%$ ethyl cyanoacetate,

$\mathrm{A}_{\mathrm{s}}$-the peak area of the internal standard benzene,

$\mathrm{A}_{\mathrm{r}}$-the peak area of $99 \%$ ethyl cyanoacetate.

The mass fraction of ethyl cyanoacetate is calculated as follows:

$$
\mathrm{m}_{\mathrm{i}}=\frac{A_{i}}{A_{s}} \bullet \frac{m_{s}}{m} \bullet f \times 100 \%
$$

$\mathrm{M}_{\mathrm{i}}$-the mass fraction of ethyl cyanoacetate $\mathrm{A}_{\mathrm{i}}$-the peak area of ethyl cyanoacetate in the sample $\mathrm{A}_{\mathrm{s}}$-the peak area of the internal standard benzene $\mathrm{m}_{\mathrm{s}}$-the mass of the internal standard benzene.

$\mathrm{m}_{\mathrm{t}}$-the quality of the sample

A mixture of $99 \%$ ethyl cyanoacetate with a mass ratio of 2:1 and benzene was injected to obtain a peak spectrum, and correction factors were calculated, and three sets of data were performed to obtain an average value of the correction factors.

Each sample was tested three times, and three peak graphs were obtained, and the average value was calculated to obtain the content of ethyl cyanoacetate in the sample, thus obtaining the esterification rate.

\subsection{Gas chromatography analysis}

According to the detection of gas chromatograph, the retention time of absolute ethanol was $0.50 \mathrm{~min}$, the retention time of internal standard substance was $0.57 \mathrm{~min}$, and the retention time of cyanoacetate was $0.80 \mathrm{~min}$

\subsubsection{Qualitative analysis}

The calculation formula of correction factor $f$ is Formula 1.The mass of benzene was $1.12 \mathrm{~g}$, the mass of $99 \%$ ethyl cyanoacetate was $2.24 \mathrm{~g}$, and the mass ratio was $1: 2$. The first correction factor $\mathrm{f}$ is calculated as follow :

$$
\mathrm{f}=\frac{\mathrm{m}_{\mathrm{r}} / \mathrm{m}_{\mathrm{s}}}{A_{r} / A_{s}}=\frac{m_{r}}{m_{s}} \times \frac{A_{s}}{A_{r}}=2 \times(56.2 \div 39.4)=2.85
$$

The chromatogram of the first correction factor is shown in Figure 2, and table(1) is description of gas chromatogram .

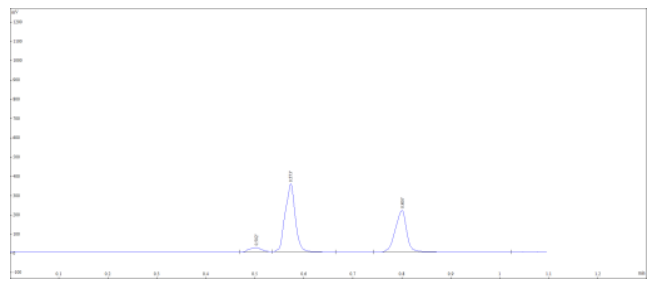

Fig. 2 chromatogram

Table(1 ) Gas chromatogram description

\begin{tabular}{lcll}
\hline & & \\
NO. Retention time & Peak area \% & Peak area \\
\hline 1 & 0.502 & 4.373 & 39008 \\
2 & 0.573 & 56.23 & 501528 \\
3 & 0.800 & 39.4 & 351413 \\
\hline \multicolumn{2}{l}{ total } & 100 & 891949 \\
\hline
\end{tabular}

The second correction factor is calculated:

$$
\mathrm{f}=\frac{\mathrm{m}_{\mathrm{r}} / \mathrm{m}_{\mathrm{s}}}{A_{r} / A_{s}}=\frac{m_{r}}{m_{s}} \times \frac{A_{s}}{A_{r}}=2 \times(57.9 \div 38.9)=2.97
$$

In both experiments, there will be a peak diagram of ethanol, and the absolute ethanol should be residual, but there is no influence on the experimental results and will not affect the results of the correction factor.

According to the above data, results of data are shown in Table1.

Table1 Correction factor data

\begin{tabular}{ccl}
\hline No. & Correction factor & Average value \\
\hline 1 & 2.85 & \\
2 & 2.97 & 2.90 \\
3 & 2.89 & \\
\hline
\end{tabular}

From the above algorithm, it can be seen that the correction factor is obtained when the quality ratio is $1: 1$, results are shown in Table 2.

Table 2 Correction factor data

\begin{tabular}{ccc}
\hline No. & Correction factor & Average value \\
\hline 1 & 2.94 & \\
2 & 2.83 & 2.88 \\
3 & 2.87 & \\
\hline
\end{tabular}

\subsection{Calculation of esterification rate}

Fig. 3 is a gas chromatogram using mixed catalyst .Taking Fig.3 as an example and table(2) is description of gas chromatogram. 


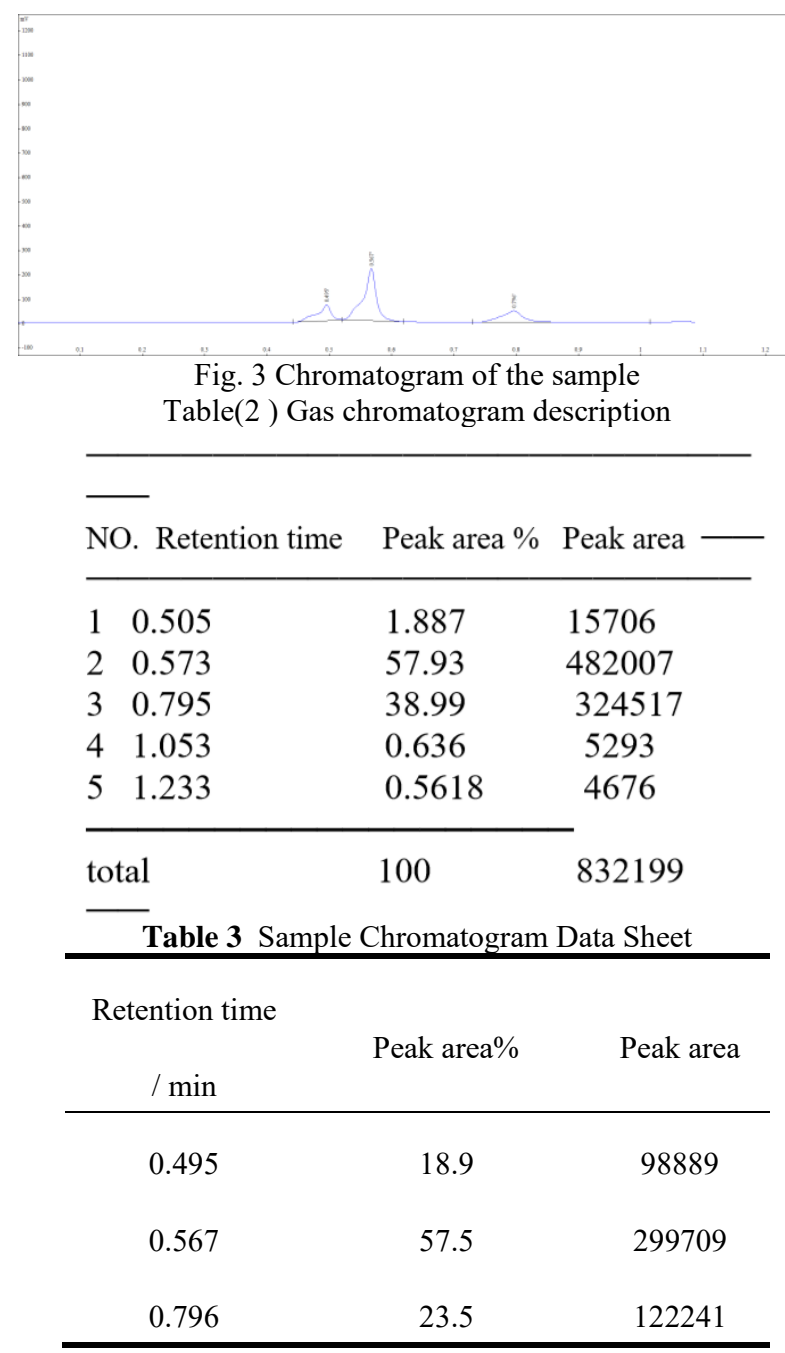

The ms:m is $2: 1$, and the mass fraction calculationis as shown in formula 5 .

$$
\begin{aligned}
& \mathrm{m}_{\mathrm{i}}=\frac{A_{i}}{A_{s}} \times \frac{m_{s}}{m} \times f \times 100 \% \\
= & 23.5 \div 57.5 \times 0.5 \times 3.74 \times 100 \% \\
= & 76.5 \%
\end{aligned}
$$

The esterification products were analyzed by gas chromatography, and the esterification rate was calculated by internal standard method.

\subsection{Analysis of esterification results}

Single-factor experiment was carried out to investigate the type of catalyst, dosage of catalyst, reaction time, molar ratio of cyanoacetic acid to absolute ethanol, and reaction temperature respectively.

\subsubsection{Orthogonal experimental results}

According to the single factor experiment, the orthogonal experiment with four factors and three levels was used to determine the optimum reaction conditions. The table of factor levels is shown in Table 4 , and the experimental result is shown in Table5.
Table4 Orthogonal empirical factors table

\begin{tabular}{ccccc}
\hline & $\begin{array}{c}\text { Molar } \\
\text { ratio } \\
(\mathrm{A}) /\end{array}$ & Time (B) $/ \mathrm{h}$ & $\begin{array}{c}\text { Tempera } \\
\text {-ture } \\
\text { (C) }\end{array}$ & $\begin{array}{c}\text { Amount of } \\
\text { catalyst used } \\
(\mathrm{n})\end{array}$ \\
\hline 1 & $1: 2.5$ & 3 & 80 & $0.5 \%$ \\
2 & $1: 3$ & 3.5 & 85 & $1 \%$ \\
3 & $1: 3.5$ & 4 & 90 & $1.5 \%$ \\
\hline
\end{tabular}

As known from orthogonal experiments. The larger $\mathrm{R}$ means that this factor has the greatest influence on the experimental results, and this factor is also very important.

From the above, it can be seen that the most influential factor on the esterification rate of ethyl cyanoacetate is the amount of catalyst, followed by the molar ratio, temperature, and reaction time.

The optimum reaction conditions were 1:3.5 molar ratio of cyanoacetic acid and absolute ethanol, $3.5 \mathrm{~h}$ reaction time, $80{ }^{\circ} \mathrm{C}$ reaction temperature and $1.5 \%$ catalyst. The esterification rate of ethyl cyanoacetate obtained under this reaction condition was the highest.

The chromatogram of the optimum reaction conditions is shown in Figure 3 and table(3) is

\begin{tabular}{|c|c|c|c|c|c|}
\hline \multirow{2}{*}{ No. } & \multicolumn{4}{|c|}{ Factors } & \multirow{2}{*}{$\begin{array}{l}\text { Esterificati } \\
\text {-on rate } \%\end{array}$} \\
\hline & A & B & $\mathrm{C}$ & $\mathrm{D}$ & \\
\hline 1 & 1 & 1 & 1 & 1 & 71.1 \\
\hline 2 & 1 & 2 & 2 & 2 & 73.2 \\
\hline 3 & 1 & 3 & 3 & 3 & 80.5 \\
\hline 4 & 2 & 1 & 2 & 3 & 85.2 \\
\hline 5 & 2 & 2 & 3 & 1 & 77.1 \\
\hline 6 & 2 & 3 & 1 & 2 & 80.5 \\
\hline 7 & 3 & 1 & 3 & 2 & 81.3 \\
\hline 8 & 3 & 2 & 1 & 3 & 91.5 \\
\hline 9 & 3 & 3 & 2 & 1 & 76.5 \\
\hline $\mathrm{k}_{1}$ & 0.75 & 0.79 & 0.82 & 0.75 & \\
\hline $\mathrm{k}_{2}$ & 0.81 & 0.81 & 0.78 & 0.78 & \\
\hline $\mathrm{k}_{3}$ & 0.83 & 0.80 & 0.80 & 0.86 & \\
\hline \multirow[t]{2}{*}{$\mathrm{R}$} & 0.08 & 0.02 & 0.04 & 0.11 & \\
\hline & $\mathrm{A}_{3}$ & $\mathrm{~B}_{2}$ & $\mathrm{C}_{1}$ & $\mathrm{D}_{3}$ & \\
\hline $\begin{array}{l}\text { Optimum } \\
\text { condition }\end{array}$ & \multicolumn{5}{|c|}{$\mathrm{A}_{3} \mathrm{~B}_{2} \mathrm{C}_{1} \mathrm{D}_{3}$} \\
\hline
\end{tabular}
description of gas chromatogram .

Table 5 Orthogonal empirical results and analysis

Fig.4 Chromatogram of optimum reaction conditions 
Table(3 ) Gas chromatogram description

\begin{tabular}{|c|c|c|c|}
\hline NO. & Retention time & e $\quad$ Peak area $\%$ & Peak area \\
\hline 1 & 0.165 & 0.007241 & 71 \\
\hline 2 & 0.284 & 0.004478 & 44 \\
\hline 3 & 0.375 & 0.1555 & 1523 \\
\hline 4 & 0.448 & 0.1473 & 1444 \\
\hline 5 & 0.503 & 23.35 & 228749 \\
\hline 6 & 0.574 & 50.79 & 497620 \\
\hline 7 & 0.728 & 0.0475 & 465 \\
\hline 8 & 0.802 & 23.96 & 234735 \\
\hline tota & & 100 & 964651 \\
\hline
\end{tabular}

\section{Conclusions}

The catalyst used in the esterification of cyanoacetic acid and absolute ethanol was determined to be the same proportion of silicotungstic acid and p-toluene sulfonic acid.

It is found that the most important factor affecting the esterification rate of ethyl cyanoacetate is the amount of catalyst, followed by the molar ratio, esterification temperature and esterification time by analysis of orthogonal experiments, and the optimum reaction conditions are determined by orthogonal experiments as follows: The molar ratio of cyanoacetic acid and absolute ethanol was $1: 3.5$, the reaction time was $3.5 \mathrm{~h}$, the reaction temperature was $80{ }^{\circ} \mathrm{C}$ and the amount of catalyst was $1.5 \%$.

The esterification rate of optimum conditions was analyzed by gas chromatograph and calculated by internal standard method. The esterification rate was $91.5 \%$.

\section{Acknowledgments}

Thanks to the guidance program of Liaoning Key Laboratory of Chemical Additive Synthesis and Separation.

\section{References}

1. $\mathrm{S}$ jian, $C$ lina, $Z$ ye.Fine Petrochemical ,29, 48 (2012)

2. $\mathrm{L} \quad \mathrm{xu}, \mathrm{Y}$ guohua, $\mathrm{W}$ jinhua.Fine and special chemicals, 24,35(2016)

3. D Pascale, C Jacques. Applied Catalysis A,129,217(1995)

4. K M Parida, M Sujata. Journal of Molecular Catalyst A: Chemical,275,77(2007) 